\title{
Cardiac Progenitor Cells: Hiding in Plain Sight
}

\author{
Mark Sussman,
}

\section{San Diego State University, San Diego, CA}

The field of cardiac biology has been revolutionized by the discovery of stem cells capable of contributing to the repair and regeneration of myocardial tissue following pathologic damage. Identification and tracking of these cells in the heart has been hampered by a lack of specific probes unique to the myocardium and controversies related to the use of markers traditionally associated with cells of the hematopoetic stem cell lineage. The unambiguous identification and tracking of these cells is essential for future studies designed to elucidate the biology of cardiac stem cells and manipulate their function to enhance regenerative processes. Reproducibility and accuracy in identification of the myocardial stem cell population is critical for the advancement of this research.

Our experience with detection of myocardial stem cells indicates that these are not trivial protocols and require investment of both time and thought on the part of the investigator. The cells are difficult to identify for the uninitiated and varying degrees of success will inevitably result depending upon immunolabeling protocols, detection systems, and expertise. We have employed fluorescence amplification protocols to enhance our signal:noise ratio and ferret out low level signals from myocardial sections. Persistence and perseverance has allowed our laboratory to verify that cardiac stem cells are present and respond to various cardioactive stimuli. Confocal microscopy performed with markers for proliferating cells (Ki67), and cardiomyocytes (GATA) reveals increased numbers of proliferating cardiomyocytes in the postnatal heart that declines within the first few weeks after birth. Cardiac progenitor populations can also be observed in the border zone surrounding infarcts in mice as well as isolated niche compartments throughout the healthy myocardium. While c-kit is an excellent marker for cardiac progenitor cells, Sca-1 shows promiscuous cross reactivity with endothelial cells of the vasculature throughout the myocardium and can only be used to identify stem cells in conjunction with another concurrent label to distinguish the endothelium from other cells in the heart.

Approximately $16 \%$ of cardiomyocyte nuclei express Ki67 at two days after birth, declining four fold by two weeks after birth to only 4\%. One week later at postnatal week 3 the number of cardiomyocytes expressing Ki67 drops to 2\%. These levels of cardiomyocyte proliferative marker expression were compared with a transgenic line expressing cardiomyocyte-specific nuclear-targeted Akt that enhances cell survival and expands the stem cell pool approximately two fold as assessed by c-kit labeling in the adult myocardium. Whereas the percentage of Ki67 expressing cardiomyocytes is comparable between normal and transgenic mouse hearts at postnatal day 2, the number of Ki67 positive cardiomyocytes is 2.2 fold higher at two weeks after birth in the transgenic hearts and remains elevated at three weeks after birth. The persistent presence of these cycling myocytes may account for the phenotypic characteristics of the nucleartargeted Akt hearts, which possess more numerous but smaller myocytes than normal hearts. Ongoing experiments with markers for stem cells (c-kit, sca-1) in the myocardium 
show similar findings for stem cells in both the normal and nuclear-targeted Akt mice. These results demonstrate that nuclear-targeting of Akt enhances cardiomyocyte proliferation and expands the number of stem cells in the myocardium of transgenic mice.

A

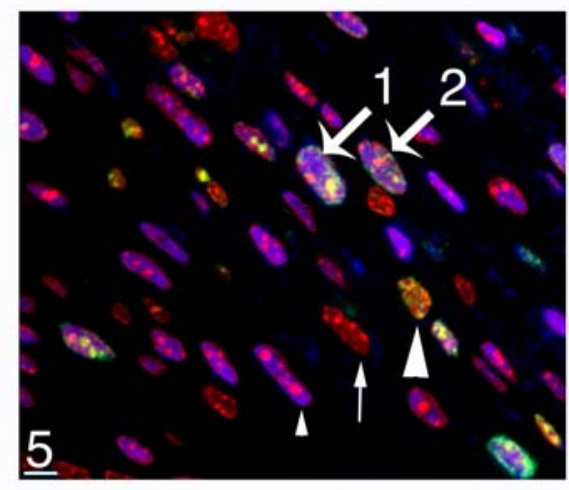

C
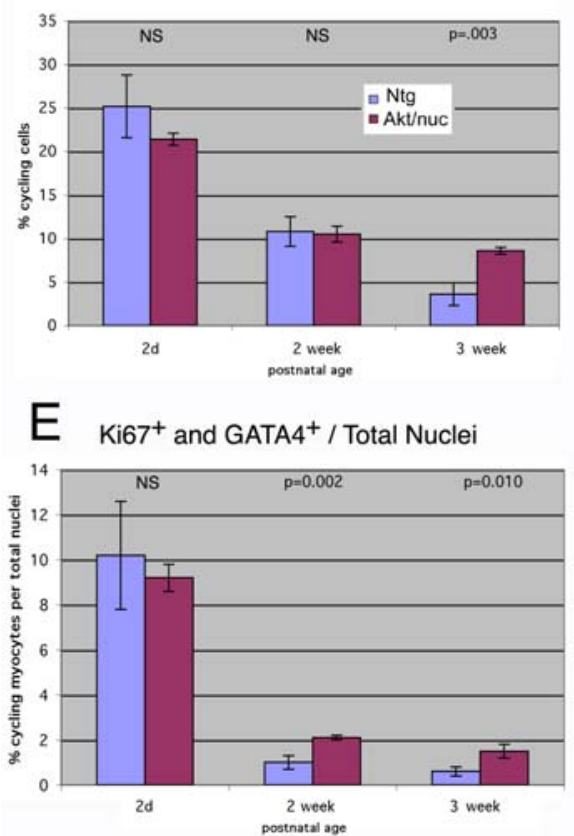

B

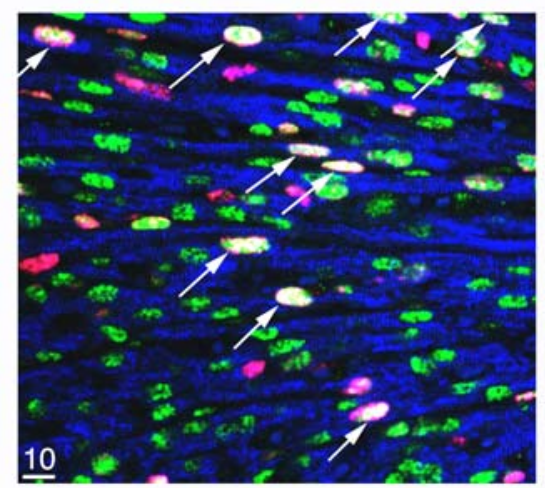

D

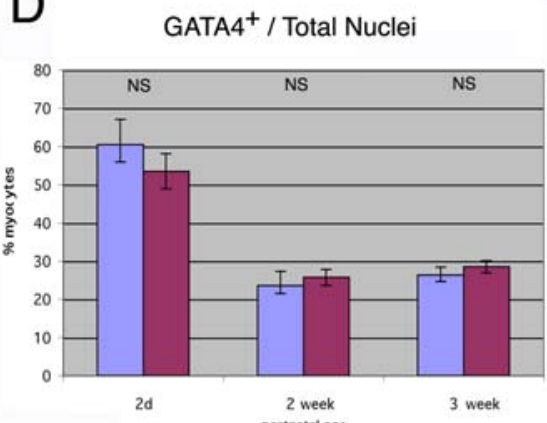

F $\quad \mathrm{Ki} 7^{+}$and $\mathrm{GATA}^{+}{ }^{+} \mathrm{GATA}^{+}$

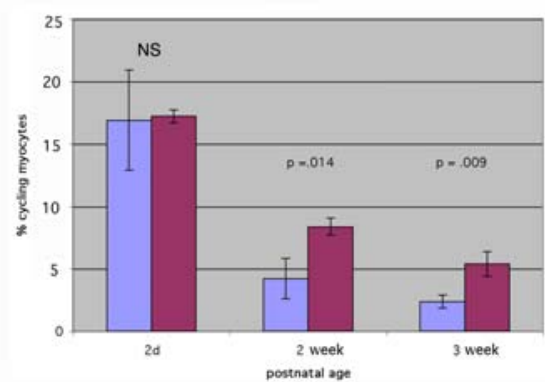

Figure 2. Differences in $\mathrm{Ki}^{+} 7^{+}$and $\mathrm{GATA4}^{+}$cell populations during postnatal development of normal and Akt/nuc hearts. Representative immunolabeling from postnatal day 2 Akt/nuc myocardium sections (A, B) and quantitation (C-F) of $\mathrm{Ki}^{+} 7^{+}$and GATA4 $4^{+}$nuclei in normal versus Akt/nuc myocardium as a function of postnatal development. A, Small field confocal scan showing nuclear labeling for Ki67 (green), GATA4 (blue), and total nuclei (red). Indicated nuclei show absence of Ki67 or GATA4 (small arrow), GATA4 alone (small arrowhead), Ki67 alone (large arrowhead), and Ki67 together with GATA4 (wide numbered arrows). B, Wide field confocal scan showing nuclear labeling for Ki67 (red) and GATA4 (green) together with the sarcomeric marker desmin (blue). Indicated nuclei show coincidence of Ki67 together with GATA4. Scale bar as indicated in bottom left for each micrograph. C-F, Quantitation of cells in the ventricular myocardium for Ki67+ / Total Nuclei (C), GATA4 ${ }^{+}$/ Total Nuclei (D), $\mathrm{Ki}_{6} 7^{+}$and $\mathrm{GATA}_{4}^{+} /$Total Nuclei (E), and Ki67 ${ }^{+}$and $\mathrm{GATA}^{+} / \mathrm{GATA}^{+}$Nuclei (F). Statistical significance values as indicated above bars, NS - not significant. 